\title{
Neuroimaging to Investigate Multisystem Involvement and Provide Biomarkers in Amyotrophic Lateral Sclerosis
}

\author{
Pierre-François Pradat ${ }^{1,2,3,4}$ and Mohamed-Mounir El Mendili ${ }^{1,2,3}$ \\ ${ }^{1}$ Sorbonne Universités, UPMC Universités Paris 06, UMR 7371, UMR-S 1146, LIB, 75005 Paris, France \\ ${ }^{2}$ CNRS, UMR 7371, LIB, 75005 Paris, France \\ ${ }^{3}$ INSERM, UMR-S 1146, LIB, 75005 Paris, France \\ ${ }^{4}$ Département des Maladies du Système Nerveux, Hôpital Pitié-Salpêtrière (AP-HP), 75005 Paris, France
}

Correspondence should be addressed to Pierre-François Pradat; pierre-francois.pradat@psl.aphp.fr

Received 21 February 2014; Accepted 25 March 2014; Published 17 April 2014

Academic Editor: Ana Cristina Calvo

Copyright ( $) 2014$ P.-F. Pradat and M.-M. El Mendili. This is an open access article distributed under the Creative Commons Attribution License, which permits unrestricted use, distribution, and reproduction in any medium, provided the original work is properly cited.

\begin{abstract}
Neuroimaging allows investigating the extent of neurological systems degeneration in amyotrophic lateral sclerosis (ALS). Advanced MRI methods can detect changes related to the degeneration of upper motor neurons but have also demonstrated the participation of other systems such as the sensory system or basal ganglia, demonstrating in vivo that ALS is a multisystem disorder. Structural and functional imaging also allows studying dysfunction of brain areas associated with cognitive signs. From a biomarker perspective, numerous studies using diffusion tensor imaging showed a decrease of fractional anisotropy in the intracranial portion of the corticospinal tract but its diagnostic value at the individual level remains limited. A multiparametric approach will be required to use MRI in the diagnostic workup of ALS. A promising avenue is the new methodological developments of spinal cord imaging that has the advantage to investigate the two motor system components that are involved in ALS, that is, the lower and upper motor neuron. For all neuroimaging modalities, due to the intrinsic heterogeneity of ALS, larger pooled banks of images with standardized image acquisition and analysis procedures are needed. In this paper, we will review the main findings obtained with MRI, PET, SPECT, and nuclear magnetic resonance spectroscopy in ALS.
\end{abstract}

\section{Introduction}

Amyotrophic lateral sclerosis (ALS) is a neurodegenerative disease characterized by a progressive alteration in the upper, or cortical, motor neurons and lower motor neurons, located in the spinal cord and brainstem. Due to its clinical heterogeneity and the lack of biological markers to diagnose ALS, the delay between the first symptoms and the diagnosis is evaluated at 9-13 months [1]. There is an unmet need to find specific and early biomarkers to help to diagnose and characterize phenotype or progression [2].

Signs of central motor neuron degeneration are often difficult to detect in clinical practice, justifying the interest for objective neuroimaging markers of upper motor neuron (UMN) involvement. It has been estimated that clinical UMN signs are absent at first examination in 7 to $10 \%$ of patients who further develop full-blown ALS $[3,4]$. The diagnostic delay is increased in patients who present isolated lower motor neuron (LMN) signs [3]. It is needed to rule out other LMN pathologies such as spinal muscular atrophy (SMA), bulbospinal muscular atrophy (Kennedy disease), or multifocal neuropathies with conduction blocks [1]. The apparition of central signs during the follow-up may allow confirming the diagnosis of ALS but this is not the case in all patients, notably because the severity of peripheral signs can mask central signs.

Neuroimaging methods allow investigating in vivo the extent of neurological systems degeneration. There is a growing body of evidence demonstrating that ALS is a multisystem neurological disorder. Autopsy studies have shown that degeneration of central nervous system structures is not restricted to the primary motor cortex and the pyramidal tract [5-8]. In addition to motor signs, cognitive signs are detected by neuropsychological tests in about $50 \%$ 
of patients with sporadic ALS and typical frontotemporal dementia (FTD) occurs in approximately $10 \%$ of the patients $[9,10]$. Association of ALS and FTD occurs in the majority of C9ORF72-linked familial ALS (FALS) [11, 12]. Atypical clinical features can be associated, defining "ALS plus" [1] syndromes with signs and symptoms involving the sensory (particularly in SOD1-linked FALS) [13-22], extrapyramidal $[14,23-31]$, cerebellar [23, 32], ocular [33, 34], and autonomic [35] systems.

In this paper, we will review the main findings obtained with different modalities of neuroimaging in ALS. We will especially focus on magnetic resonance imaging (MRI) studies and particularly on spinal cord neuroimaging that has shown great developments in the last few years.

\section{Magnetic Resonance Imaging}

2.1. Conventional Magnetic Resonance Imaging. Beyond its role to exclude several "ALS-mimick" syndromes [1], abnormalities suggestive of upper motor neuron involvement are sometimes detected. Several studies, using various modalities (T2* , fluid-attenuated inversion recovery (FLAIR) or fast spin echo proton density-weighted imaging), have shown hyperintensity in the white matter along the corticospinal tract, from the centrum semiovale to the brainstem [3646]. However, such abnormalities are rare, nonspecific, not readily quantifiable, and do not correlate with disease severity or rate of progression [47]. A cortical atrophy, which is predominant in the frontal region, and a characteristic $\mathrm{T} 2{ }^{*}$ or FLAIR hypointensity at the level of the primary motor cortex have been described in the literature but are exceptionally detectable in clinical practice $[39,48]$.

2.2. Advanced MRI. Diffusion-based neuroimaging methods allow evaluating the degeneration of white matter fiber bundles. Diffusion results from the random movement of molecules in vivo. Diffusion of water in structures such as the cerebrospinal fluid (CSF) or grey matter is isotropic (identical in all directions), whereas in white matter it occurs preferentially along the axis of orientation of the fiber bundles. The application of diffusion gradients in several directions results in diffusion tensor imaging (DTI). An anisotropy map can thus be obtained, which provides information about the microstructural organization of the white matter.

A study published in 1999 demonstrated a decrease in fractional anisotropy (FA) in the intracranial portion of the corticospinal tract (subcortical white matter, internal capsule, and brainstem) [49]. It has been confirmed by numerous other studies [50-60]. Abnormalities have been observed in patients who had no UMN signs at the time of MRI investigation but developed pyramidal tract symptoms later in the course of their disease, suggesting that DTI could contribute to earlier diagnosis of ALS in patients with pure LMN involvement [51]. It has been suggested that a decreased fractional anisotropy (FA) in the corpus callosum (CC) was a good DTI marker in patients with ALS [61]. These changes may correspond to degeneration of transcallosal fibers passing between primary motor cortices. Another study showed that FA in motor-related regions of the CC is more affected than in other CC areas in ALS patients [62].

Tractography is a method based on DTI that enables reconstruction of the three-dimensional geometry of the pyramidal tract [63] and allows establishing an FA profile along the pyramidal tract [64]. A tractography study showed that the decreases in FA are largely limited to the precentral areas in patients with ALS [52]. Using tractography to segment the corticobulbar tract, lower FA was measured in patients with bulbar-onset versus limb-onset disease [65].

By using a voxel-by-voxel approach, which consists of comparing groups of patients without an a priori predefined region of interest, our team and other groups have showed that abnormalities on DTI were detectable outside the primary motor regions, thereby confirming that ALS is a multisystem degenerative disorder [53]. Diffuse lesions have also been observed using voxel-based morphometry (VBM), a technic allowing automated segmentation and quantification of grey and white matter volumes to study regional differences [66]. Recently authors used high-resolution T1weighted imaging data for model-based subcortical registration and segmentation to explore the involvement of subcortical structures [67]. They used vertex-wise statistics that provide quantitative, visual, surface-projected information about the shape of the various subcortical structures. This analysis revealed changes affecting the basal ganglia including the superior and inferior aspects of the bilateral thalami, the lateral and inferior portion of the left hippocampus, and the medial and superior aspect of the left caudate (Figure 1). The authors conclude that dysfunction of frontostriatal networks is likely to contribute to the unique neuropsychological profile of ALS, dominated by executive dysfunction, apathy, and deficits in social cognition.

The discovery that the presence of a hexanucleotide expansion in C9orf72 gene $[11,12]$ was associated with FTD in ALS led to specific neuroimaging investigations. A recent study used tract-based spatial statistics of multiple white matter diffusion parameters, cortical thickness measurements, and VBM analyses in C9orf72-negative patients with ALS carrying the C9orf72 hexanucleotide repeat expansion [68]. The result is that extensive cortical and subcortical frontotemporal involvement was identified in association with the C9orf72 genotype, compared to the relatively limited extramotor pathology in patients with C9orf72-negative ALS [67]. In contrast, a DTI study showed that patients with SOD1 gene-linked FALS showed less extensive pathologic white matter in motor and extramotor pathways compared with patients with sporadic ALS [69].

In clinical practice, the diagnostic value of FA measurement remains limited mainly because of the overlap between the values measured in ALS patients and those in control subjects. In one study, measurement of FA in the internal capsule to detect central motor neuron lesions compared with healthy subjects had a sensitivity of $95 \%$, but the specificity was only $71 \%$, with a positive predictive value of $82 \%$ [46]. A recent individual patient data (IPD) meta-analysis using corticospinal tract data suggested that the diagnostic accuracy of DTI lacks sufficient discrimination [59]. Of 30 identified studies, 11 corresponding authors provided IPD 


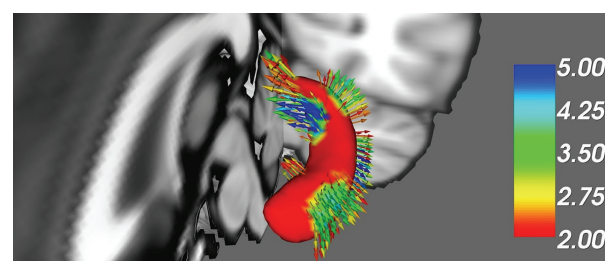

(a)

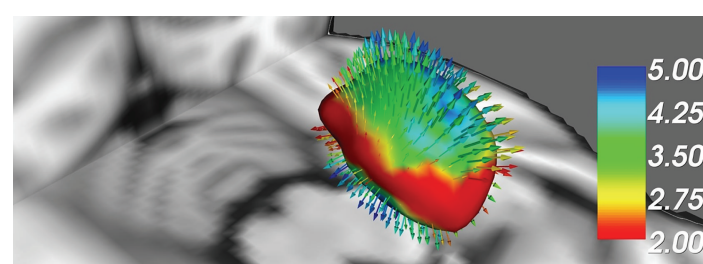

(b)

FIGURE 1: Evidence of hippocampal and basal ganglia involvement in amyotrophic lateral sclerosis. Comparative surface-based vertex analyses between healthy controls and C9orf72 hexanucleotide repeat negative ALS patients corrected for age and multiple comparisons reveal significant left hippocampal (a) and thalamic changes (b) (Courtesy of Peter Bede-Trinity College Dublin).

and 221 ALS patients and 187 healthy control subjects were available for the study. The pooled sensitivity was 0.68 (95\% CI: $0.62-0.75$ ), and the pooled specificity was 0.73 (95\% CI: $0.66-0.80)$.

2.3. Spinal Cord Imaging. Spinal MRI has the advantage to investigate the two motor system components that are involved in ALS, that is, the lower and upper motor neuron. Although widely applied to the brain, advanced methods such as DTI are challenging at the spinal level because of (i) the small size of the cord relative to the brain $(\sim 1 \mathrm{~cm}$ diameter in the human) requiring higher spatial resolution and thus decreasing the signal-to-noise ratio, (ii) physiological motions (respiration, cardiac) that may bias anisotropic diffusion coefficient estimation and create ghosting artifacts [70-72], (iii) partial volume effects that are more problematic in the cord due to the surrounding cerebrospinal fluid [73], (iv) chemical-shift artifacts arising from the epidural fat and other nearby structures, and (v) geometric distortions arising from magnetic field inhomogeneities in nearby intervertebral disks and lungs. The latter point is particularly challenging in diffusion MRI since usual sequences based on echo planar imaging (EPI) are very sensitive to such artifacts [74, 75]. In past years, with the development of new methods, such as cardiac and respiratory gating [75-78], it has been shown that DTI and magnetization transfer (MT) imaging were feasible to detect changes in the spinal cord in ALS [79-81]. In a recent study, we have shown that abnormalities in the spinal cord using a multiparametric MRI approach combing DTI, MT ratio (MTR), and atrophy measurements correlated with functional impairment [79]. In this study, local spinal cord atrophy was correlated with muscle deficits and with the motor evoked potential amplitude measured by transcranial magnetic stimulation (TMS), an index of LMN dysfunction. It suggests that regional atrophy is a sensitive biomarker of motor neuron loss in the anterior horns of the spinal cord.

Conversely, DTI and MTR changes in the corticospinal tract correlated with the higher facilitation motor threshold measured by TMS, a parameter that reflects the functionality of the pyramidal tract. Interestingly, changes of DTI metrics demonstrated a subclinical involvement of sensory pathways.

Recently, new acquisition strategies have been proposed to overcome the inherent difficulties in diffusion-weighted imaging of the spinal cord. A promising high-resolution
DWI sequence (Syngo RESOLVE) has been proposed [82]. The RESOLVE sequence allows minimization of susceptibility distortions and $\mathrm{T} 2{ }^{*}$ blurring. Furthermore, it can be combined with other acquisition strategies such as reduction field-of-view (rFOV) [83-85] and parallel imaging [86] to provide fiber tractography in large portions of the spinal cord. Such advances open doors to an accurate quantification of DTI metrics profile along the corticospinal tract or sensory tracts. This is fundamentally needed to clarify some physiopathological aspects of ALS disease, for instance, the dying-back versus dying-forward hypotheses and the possible sensory afferents involvement [79]. In parallel, the new generation of $3 \mathrm{~T}$ MRI scanners equipped with $300 \mathrm{mT} / \mathrm{m}$ gradients $[87,88]$ provides new exploratory dimensions for white matter microstructures in the spinal cord $[89,90]$. As showed in vivo as well as ex vivo for the brain, the new MRI scanners improved tissues sensitivity, signal-to-noise ratio (SNR), and spatial and angular diffusion resolution in practical time.

MRI pathological spinal cord studies were mainly focused on white matter integrity where grey matter was not for a great interest until the past few years. This was mainly related to the difficulties in imaging the spinal cord due to low SNR as well as contrastdifference between CSF and white and grey matter at $1.5 \mathrm{~T}$. Passing to higher magnetic field strength ( $3 \mathrm{~T}$ and $7 \mathrm{~T}$ ) [91-93], the construction of adapted coils for spinal cord imaging [94-96] and the adaptation of existing sequences $[91,97]$ bring new horizons for spinal cord anatomical explorations. Three recent studies showed the feasibility of white/grey matter imaging and presented reliable tools for anatomical structures characterization in controls $[98,99]$ and multiple sclerosis patients [100] using 3 T MRI-systems. In parallel, one study has showed preliminary results at $3 \mathrm{~T}$ of the construction of a probabilistic atlas and anatomical template of the human cervical and thoracic spinal cord that included CSF and white and gray matter [97]. Such atlas can be used for atrophy localization in ALS patients using VBM by adapting the methodology proposed in [101] to the case of 3 T MR images (Figure 2).

Such advances bring new perspectives for neurodegenerative diseases and particularly for ALS. Quantifications of white and grey matter degeneration are currently feasible. MRI sequences, image processing, and statistical tools have been already developed $[91,97,101-103]$ and are almost ready 


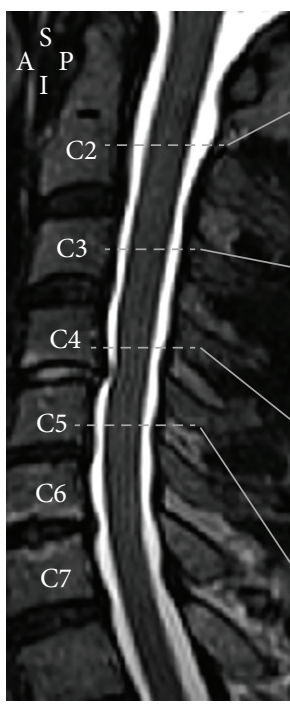

(a)
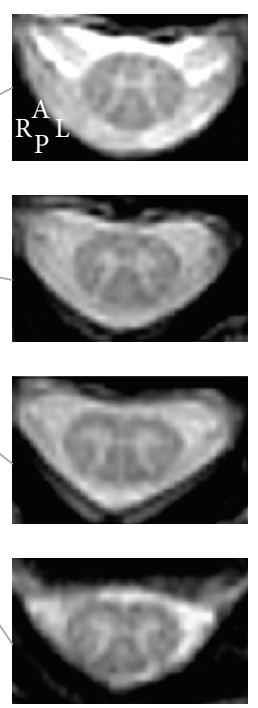

(b)
FIGURE 2: (a) T2-weighted turbo spin echo midsagittal section in a healthy subject (male, 62 years old), showing the anatomical landmarks of the cervical spinal cord. (b) $\mathrm{T} 2{ }^{*}$-weighted $2 \mathrm{D}$ gradient recalled echo axial sections at the vertebral levels $\mathrm{C} 2, \mathrm{C} 3, \mathrm{C} 4$, and $\mathrm{C} 5$ for the same subject (voxel size $=0.7 \times 0.7 \times 3 \mathrm{~mm}$ ). Images have been acquired using a 3 T MRI system (TIM Trio 32-channel, Siemens Healthcare, Erlangen, Germany). A: anterior; I: inferior; L: left; P: posterior; R: right; S: superior.

for use to figure out some of the mechanisms involved in spinal cord tissues degeneration in ALS.

2.4. Functional MRI. Functional MRI (fMRI), by measuring cortical blood oxygen level dependent (BOLD) signal changes, provides a tool to study cortical function and reorganization. Regional modifications in cerebral blood flow have been studied during hand motor tasks [104]. Several studies have demonstrated that cerebral activation involved more extensive cortical regions than in control subjects. However, whether it reflects cortical reorganization [104] or it is the result of cortical functional adaptation due to peripheral weakness [105] remains a matter of debate. Using a simple hand motor task when the motor deficit is still moderate, a study showed that cerebral activation is correlated with the rate of disease progression suggesting that brain functional rearrangement in ALS may have prognostic implications [106].

Unlike the traditional fMRI, the recently developed resting-state functional (rfMRI) techniques avoid potential performance since rfMRI does not require the subjects to perform any task $[107,108]$. Studies that have been carried out in ALS provide abundant evidence for a reorganisation of various cerebral networks [108-113]. Interestingly, one study showed that rfMRI changes correlated with the rate of disease progression and duration [114].

\section{Nuclear Magnetic Resonance Spectroscopy}

Nuclear magnetic resonance spectroscopy (NMRS) allows measuring the neurochemical profile of a particular region of the brain in vivo. The main peak is $\mathrm{N}$-acetylaspartate (NAA), which is considered as a marker of neuronal integrity. Several studies have demonstrated a decrease in NAA [115, 116] and/or ratios of NAA with choline-containing compounds (Cho) and creatine (Cr) [NAA/Cho and NAA/Cr ratios] at the level of the motor cortex of patients with ALS $[115,117]$.

For diagnosis applications, studies suggested that NMRS spectroscopy may discriminate between patients with classical ALS and patients with progressive muscular atrophy, a disease confined to LMNs $[118,119]$. The diagnostic value is limited because of an overlap between those values in ALS patients and healthy controls. It has been suggested that the combination of $\mathrm{N}$-acetylaspartate (NAA) and myo-inositol may improve the specificity of the test but further studies are needed [120]. Using a whole-brain resonance spectroscopic imaging approach, NAA showed a significant relationship with disability [121]. A cross-sectional study assessing proton NMRS of the cervical spine showed that NAA/Cr and NAA/Myo ratios were reduced in patients with ALS but also in SOD1-positive people at risk for FALS, suggesting that neurometabolic changes occur early in the course of the disease process [122]. Recent advances in high resolution NMRS at $3 \mathrm{~T}$ allow direct quantification of GABA in the cortex [123]. Recently, a study in a small number of subjects showed that decreased levels of GABA were present in the motor cortex of ALS patients compared to healthy controls [124]. It suggests that a loss of inhibition by interneurons may play a role in neurodegeneration through excitotoxicity mechanisms.

\section{Positron Emission Tomography}

Positron emission tomography (PET) and monophotonic emission tomography (single photon emission computerized tomography (SPECT)) are nuclear imaging techniques which use various tracers to either reveal neuron dysfunction or investigate a pathogenic mechanism involved in the disease. Using SPECT, several studies have demonstrated a decrease in cerebral blood flow after injection of hexamethyl-propyleneamine oxime labelled with technetium-99 m (99 mTc). They showed abnormalities in the primary motor cortex [125-128], which can also extend in an anterior fashion into the frontal lobes, particularly in patients with associated cognitive problems [129]. Studies using PET with 2-fluoro-2-deoxy-glucose also showed a variable decrease in cerebral glucose metabolism at rest [130132]. Interestingly, a PET study found not only hypometabolic but also hypermetabolic areas in the brain of sporadic ALS patients, possibly due to increased FDG uptake by astrocytes and/or microglia [133]. In a recent study, the authors report an FDG metabolism study in patients with the C9orf72 mutation compared to nonmutated ALS patients (either with or without dementia) [134]. The conclusion is that C9orf72 
mutated ALS patients t have a more widespread central nervous system involvement than ALS patients without genetic mutations, with or without dementia.

A PET study showed a widespread loss of binding of the GABAA ligand $\left[{ }^{11} \mathrm{C}\right]$-flumazenil in sporadic ALS patients [135]. Because GABAA receptors are widely distributed in the cerebral cortex and are located both on pyramidal cells and interneurons, $\left[{ }^{11} \mathrm{C}\right]$-flumazenil provides a means of detecting motor and extramotor dysfunction in ALS. It was shown that abnormalities were less extensive in patients with SOD1linked familial ALS patients, suggesting that GABA-ergic neurotransmission may be less severely impaired in these cases [136]. Nigrostriatal dysfunction has also been shown by both PET [137] and SPECT studies [138].

PET imaging can also detect inflammatory processes that are implicated in the pathogenesis of ALS. Assessment of microglial activation can be performed through neuroimaging of the $18 \mathrm{kDA}$ translocator protein (TSPO) that is present in activated glial cells, using selective TSPOselective radioligands such as ${ }^{11} \mathrm{C}-\mathrm{PK} 11195$. Extensive cortical abnormalities in fixation of this tracer, predominantly in the frontal and temporal lobes have been observed in one study [139]. The DPA714 radioligand has a longer half-life, a better bioavailability, and less nonspecific binding than PK11195 [140]. This allows the examination of milder microglial activation by PET. A prospective study showed significant binding of DPA714 both in motor cortex areas and also in temporal areas since the earlier stages of the disease [141]. With the continuing development in new radioligands, PET imaging may potentially provide tools to monitor the effect of drugs targeting inflammation.

\section{Conclusion}

In the recent years, thanks to technological and methodological developments, neuroimaging was revealed as an indispensable research tool to understand the pathophysiology of ALS. Because ALS is a multisystem disorder and not a pure motor neuron disease, there is a need to investigate in vivo the participation of other systems such as the sensory or extrapyramidal systems. Neuroimaging studies have already provided insights about the potential role of sensory feedback, inflammation, and loss of inhibition by interneurons in the pathogenesis of ALS. Studies on presymptomatic carriers of mutations responsible for FALS provide the unique opportunity to study the very early mechanism that triggers the neurodegenerative cascade leading to the loss of motor neurons.

Among MRI techniques, although DTI initially appeared as the most promising diagnostic tool, the disappointing results of a meta-analysis suggest that a multiparametric approach will be required to make neuroimaging a critical component in the workup of ALS. Development of spinal cord imaging will be a key element to provide useful biomarkers as sensitive and specific as possible to help diagnosis and to characterize phenotype or progression. Due to the intrinsic heterogeneity of ALS, larger pooled banks of images with standardized image acquisition and analysis procedures are needed. For this purpose, the NeuroImaging Society in ALS (NISALS) has emerged in 2010 and has an interactive web Platform (http://nedigs05.nedig.uni-jena.de/nisals/) to provide quality controlled MRI data for the international scientific community.

\section{Conflict of Interests}

The authors declare that there is no conflict of interests regarding the publication of this paper.

\section{Acknowledgments}

The authors thank the French Association for Myopathies (AFM), the Institute for Research on Spinal Cord and Brain (IRME), and IHU-A-ICM via the program "Investissements d'avenir" (ANR-10-IAIHU-06) for their research support to the cited studies performed by the authors of the paper.

\section{References}

[1] P.-F. Pradat and G. Bruneteau, "Differential diagnosis and atypical subsets of ALS," Revue Neurologique, vol. 162, no. 2, pp. S81-S90, 2006.

[2] P.-F. Pradat and M. Dib, "Biomarkers in amyotrophic lateral sclerosis: facts and future horizons," Molecular Diagnosis \& Therapy, vol. 13, no. 2, pp. 115-125, 2009.

[3] S. Zoccolella, E. Beghi, G. Palagano et al., "Predictors of delay in the diagnosis and clinical trial entry of amyotrophic lateral sclerosis patients: a population-based study," Journal of the Neurological Sciences, vol. 250, no. 1-2, pp. 45-49, 2006.

[4] B. J. Traynor, M. B. Codd, B. Corr, C. Forde, E. Frost, and O. M. Hardiman, "Clinical features of amyotrophic lateral sclerosis according to the El Escorial and Airlie House diagnostic criteria: a population-based study," Archives of Neurology, vol. 57, no. 8, pp. 1171-1176, 2000.

[5] K. Iwanaga, S. Hayashi, M. Oyake et al., "Neuropathology of sporadic amyotrophic lateral sclerosis of long duration," Journal of the Neurological Sciences, vol. 146, no. 2, pp. 139-143, 1997.

[6] P. Castaigne, F. Lhermitte, J. Cambier, R. Escourolle, and P. le Bigot, "Etude neuropathologique de 61 observations de sclérose latérale amyotrophique. Discussion nosologique," Revue Neurologique, vol. 127, no. 4, pp. 1-414, 1972.

[7] S. Sasaki, Y. Tsutsumi, K. Yamane, H. Sakuma, and S. Maruyama, "Sporadic amyotrophic lateral sclerosis with extensive neurological involvement," Acta Neuropathologica, vol. 84, no. 2, pp. 211-215, 1992.

[8] Y. Kawamura, P. J. Dyck, and M. Shimono, "Morphometric comparison of the vulnerability of peripheral motor and sensory neurons in amyotrophic lateral sclerosis," Journal of Neuropathology and Experimental Neurology, vol. 40, no. 6, pp. 667-675, 1981.

[9] C. Lomen-Hoerth, J. Murphy, S. Langmore, J. H. Kramer, R. K. Olney, and B. Miller, "Are amyotrophic lateral sclerosis patients cognitively normal?” Neurology, vol. 60, no. 7, pp. 1094-1097, 2003.

[10] P. H. Gordon, D. Delgadillo, A. Piquard et al., "The range and clinical impact of cognitive impairment in French patients with ALS: a cross-sectional study of neuropsychological test performance," Amyotrophic Lateral Sclerosis, vol. 12, no. 5, pp. 372-378, 2011. 
[11] M. DeJesus-Hernandez, I. R. Mackenzie, B. F. Boeve et al., "Expanded GGGGCC hexanucleotide repeat in noncoding region of C9ORF72 causes chromosome 9p-linked FTD and ALS," Neuron, vol. 72, no. 2, pp. 245-256, 2011.

[12] A. E. Renton, E. Majounie, A. Waite et al., "A hexanucleotide repeat expansion in C9ORF72 is the cause of chromosome 9p21linked ALS-FTD," Neuron, vol. 72, no. 2, pp. 257-268, 2011.

[13] S. S. Gubbay, E. Kahana, N. Zilber, G. Cooper, S. Pintov, and Y. Leibowitz, "Amyotrophic lateral sclerosis. A study of its presentation and prognosis," Journal of Neurology, vol. 232, no. 5, pp. 295-300, 1985.

[14] H. Mitsumoto, D. A. Chad, and E. P. Pioro, "Clinical features: signs and symptoms," in Amyotrophic Lateral Sclerosis, H. Mitsumoto, D. A. Chad, and E. P. Pioro, Eds., pp. 47-64, FA Davis Company, Philadelphie, Pa, USA, 1998.

[15] T.-M. Li, E. Alberman, and M. Swash, "Comparison of sporadic and familial disease amongst 580 cases of motor neuron disease," Journal of Neurology Neurosurgery \& Psychiatry, vol. 51, no. 6, pp. 778-784, 1988.

[16] B. Moulard, W. Camu, A. Malafosse, M. Billiard, and M. BaldyMoulinier, "Étude clinique des formes familiales de sclérose latérale amyotrophique: revue de la littérature," Revue Neurologique, vol. 153, no. 5, pp. 314-324, 1997.

[17] K. Abe, M. Aoki, M. Ikeda, M. Watanabe, S. Hirai, and Y. Itoyama, "Clinical characteristics of familial amyotrophic lateral sclerosis with $\mathrm{Cu} / \mathrm{Zn}$ superoxide dismutase gene mutations," Journal of the Neurological Sciences, vol. 136, no. 1-2, pp. 108-116, 1996.

[18] P. M. Andersen, L. Forsgren, M. Binzer et al., "Autosomal recessive adult-onset amyohophic lateral sclerosis associated with homozygosity for Asp90Ala CuZn-superoxide dismutase mutation A clinical and genealogical study of 36 patients," Brain, vol. 119, no. 4, pp. 1153-1172, 1996.

[19] W. Camu, J. Khoris, B. Moulard et al., "Genetics of familial ALS and consequences for diagnosis," Journal of the Neurological Sciences, vol. 165, no. 1, pp. S21-S26, 1999.

[20] H. F. Jafari-Schluep, J. Khoris, V. Mayeux-Portas, C. Hand, G. Rouleau, and W. Camu, "Les anomalies du gène superoxyde dismutase 1 dans la sclérose latérale amyotrophique familiale: corrélations phénotype/génotype et implications pratiques. Lexpérience française et revue de la littérature," Revue Neurologique, vol. 160, no. 1, pp. 44-50, 2004.

[21] A. Kawata, S. Kato, H. Hayashi, and S. Hirai, "Prominent sensory and autonomic disturbances in familial amyotrophic lateral sclerosis with a Gly93Ser mutation in the SOD1 gene," Journal of the Neurological Sciences, vol. 153, no. 1, pp. 82-85, 1997.

[22] J. Khoris, B. Moulard, V. Briolotti et al., "Coexistence of dominant and recessive familial amyotrophic lateral sclerosis with the D90A Cu,Zn superoxide dismutase mutation within the same country," European Journal of Neurology, vol. 7, no. 2, pp. 207-211, 2000.

[23] R. J. Davenport, R. J. Swingler, A. M. Chancellor, and C. P. Warlow, "Avoiding false positive diagnoses of motor neuron disease: lessons from the Scottish motor neuron disease register," Journal of Neurology Neurosurgery \& Psychiatry, vol. 60, no. 2, pp. 147151, 1996.

[24] A. Eisen and D. B. Calne, "Amyotrophic lateral sclerosis, Parkinson's disease and Alzheimer's disease: phylogenetic disorders of the human neocortex sharing many characteristics," The Canadian Journal of Neurological Sciences, vol. 19, no. 1, pp. 117120, 1992.
[25] A. Verma and W. G. Bradley, "Atypical motor neuron disease and related motor syndromes," Seminars in Neurology, vol. 21, no. 2, pp. 177-187, 2001.

[26] S. Zoccolella, G. Palagano, A. Fraddosio et al., "ALS-plus: 5 cases of concomitant amyotrophic lateral sclerosis and parkinsonism," Neurological Sciences, vol. 23, no. 2, pp. S123-S124, 2002.

[27] J. Desai and M. Swash, "Extrapyramidal involvement in amyotrophic lateral sclerosis: backward falls and retropulsion," Journal of Neurology, Neurosurgery \& Psychiatry, vol. 67, no. 2, pp. 214-216, 1999.

[28] H. Miwa, M. Kajimoto, and T. Kondo, "Chorea in motor neuron disease," Movement Disorders, vol. 17, no. 6, p. 1397, 2002.

[29] P. F. Pradat, F. Salachas, L. Lacomblez et al., "Association of chorea and motor neuron disease," Movement Disorders, vol. 17, no. 2, pp. 419-420, 2002.

[30] U. I. Knirsch, R. Bachus, G. Gosztonyi, R. Zschenderlein, and A. C. Ludolph, "Clinicopathological study of atypical motor neuron disease with vertical gaze palsy and ballism," Acta Neuropathologica, vol. 100, no. 3, pp. 342-346, 2000.

[31] P.-F. Pradat, G. Bruneteau, E. Munerati et al., "Extrapyramidal stiffness in patients with amyotrophic lateral sclerosis," Movement Disorders, vol. 24, no. 14, pp. 2143-2148, 2009.

[32] N. Schimke, K. Krampfl, S. Petri, R. Dengler, and J. Bufler, "Cerebral symptoms with motor neuronal disorders: a special form of ALS-plus syndrome," Der Nervenarzt, vol. 73, no. 8, pp. 751-753, 2002.

[33] H. Hayashi, S. Kato, T. Kawada, and T. Tsubaki, "Amyotrophic lateral sclerosis: oculomotor function in patients in respirators," Neurology, vol. 37, no. 8, pp. 1431-1432, 1987.

[34] R. Sharma, S. Hicks, C. M. Berna, C. Kennard, K. Talbot, and M. R. Turner, "Oculomotor dysfunction in amyotrophic lateral sclerosis: a comprehensive review," Archives of Neurology, vol. 68, no. 7, pp. 857-861, 2011.

[35] T. Shimizu, A. Kawata, S. Kato et al., "Autonomic failure in ALS with a novel SOD1 gene mutation," Neurology, vol. 54, no. 7, pp. 1534-1537, 2000.

[36] G. Cheung, M. J. Gawal, P. W. Cooper, R. I. Farb, and L. C. Ang, "Amyotrophic lateral sclerosis: correlation of clinical and MR imaging findings," Radiology, vol. 194, no. 1, pp. 263-270, 1995.

[37] G. Comi, M. Rovaris, and L. Leocani, "Neuroimaging in amyotrophic lateral sclerosis," European Journal of Neurology, vol. 6, no. 6, pp. 629-637, 1999.

[38] D. S. Goodin, H. A. Rowley, and R. K. Olney, "Magnetic resonance imaging in amyotrophic lateral sclerosis," Annals of Neurology, vol. 23, no. 4, pp. 418-420, 1988.

[39] M. J. Hecht, F. Fellner, C. Fellner, M. J. Hilz, D. Heuss, and B. Neundörfer, "MRI-FLAIR images of the head show corticospinal tract alterations in ALS patients more frequently than T2-, T1- and proton-density-weighted images," Journal of the Neurological Sciences, vol. 186, no. 1-2, pp. 37-44, 2001.

[40] E. Hofmann, G. Ochs, A. Pelzl, and M. Warmuth-Metz, "The corticospinal tract in amyotrophic lateral sclerosis: an MRI study," Neuroradiology, vol. 40, no. 2, pp. 71-75, 1998.

[41] Y. Kato, K. Matsumura, Y. Kinosada, Y. Narita, S. Kuzuhara, and T. Nakagawa, "Detection of pyramidal tract lesions in amyotrophic lateral sclerosis with magnetization-transfer measurements," American Journal of Neuroradiology, vol. 18, no. 8, pp. 1541-1547, 1997.

[42] S. Mirowitz, K. Sartor, M. Gado, and R. Torack, "Focal signalintensity variations in the posterior internal capsule: normal MR findings and distinction from pathologic findings," Radiology, vol. 172, no. 2, pp. 535-539, 1989. 
[43] J. L. Tanabe, M. Vermathen, R. Miller, D. Gelinas, M. W. Weiner, and W. D. Rooney, "Reduced MTR in the corticospinal tract and normal T2 in amyotrophic lateral sclerosis," Magnetic Resonance Imaging, vol. 16, no. 10, pp. 1163-1169, 1998.

[44] J. W. Thorpe, I. F. Moseley, C. H. Hawkes, D. G. MacManus, W. I. McDonald, and D. H. Miller, "Brain and spinal cord MRI in motor neuron disease," Journal of Neurology Neurosurgery \& Psychiatry, vol. 61, no. 3, pp. 314-317, 1996.

[45] M. Waragai, "MRI and clinical features in amyotrophic lateral sclerosis," Neuroradiology, vol. 39, no. 12, pp. 847-851, 1997.

[46] J. M. Graham, N. Papadakis, J. Evans et al., "Diffusion tensor imaging for the assessment of upper motor neuron integrity in ALS," Neurology, vol. 63, no. 11, pp. 2111-2119, 2004.

[47] J. M. C. Winhammar, D. B. Rowe, R. D. Henderson, and M. C. Kiernan, "Assessment of disease progression in motor neuron disease," The Lancet Neurology, vol. 4, no. 4, pp. 229-238, 2005.

[48] H. Oba, T. Araki, K. Ohtomo et al., "Amyotrophic lateral sclerosis: T2 shortening in motor cortex at MR imaging," Radiology, vol. 189, no. 3, pp. 843-846, 1993.

[49] C. M. Ellis, A. Simmons, D. K. Jones et al., "Diffusion tensor MRI assesses corticospinal tract damage in ALS," Neurology, vol. 53, no. 5, p. 1051, 1999.

[50] P. Valsasina, F. Agosta, B. Benedetti et al., "Diffusion anisotropy of the cervical cord is strictly associated with disability in amyotrophic lateral sclerosis," Journal of Neurology, Neurosurgery \& Psychiatry, vol. 78, no. 5, pp. 480-484, 2007.

[51] M. Sach, G. Winkler, V. Glauche et al., "Diffusion tensor MRI of early upper motor neuron involvement in amyotrophic lateral sclerosis," Brain, vol. 127, no. 2, pp. 340-350, 2004.

[52] C. A. Sage, R. R. Peeters, A. Görner, W. Robberecht, and S. Sunaert, "Quantitative diffusion tensor imaging in amyotrophic lateral sclerosis," NeuroImage, vol. 34, no. 2, pp. 486-499, 2007.

[53] L. Thivard, P.-F. Pradat, S. Lehéricy et al., "Diffusion tensor imaging and voxel based morphometry study in amyotrophic lateral sclerosis: Relationships with motor disability," Journal of Neurology, Neurosurgery \& Psychiatry, vol. 78, no. 8, pp. 889892, 2007.

[54] H. Mitsumoto, A. M. Uluğ, S. L. Pullman et al., "Quantitative objective markers for upper and lower motor neuron dysfunction in ALS," Neurology, vol. 68, no. 17, pp. 1402-1410, 2007.

[55] J. C. T. Wong, L. Concha, C. Beaulieu, W. Johnston, P. S. Allen, and S. Kalra, "Spatial profiling of the corticospinal tract in amyotrophic lateral sclerosis using diffusion tensor imaging," Journal of Neuroimaging, vol. 17, no. 3, pp. 234-240, 2007.

[56] S. K. Schimrigk, B. Bellenberg, M. Schlüter et al., "Diffusion tensor imaging-based fractional anisotropy quantification in the corticospinal tract of patients with amyotrophic lateral sclerosis using a probabilistic mixture model," American Journal of Neuroradiology, vol. 28, no. 4, pp. 724-730, 2007.

[57] C. R. V. Blain, V. C. Williams, C. Johnston et al., "A longitudinal study of diffusion tensor MRI in ALS," Amyotrophic Lateral Sclerosis, vol. 8, no. 6, pp. 348-355, 2007.

[58] J. Li, P. Pan, W. Song, R. Huang, K. Chen, and H. Shang, "A metaanalysis of diffusion tensor imaging studies in amyotrophic lateral sclerosis," Neurobiology of Aging, vol. 33, no. 8, pp. 18331838, 2011.

[59] B. R. Foerster, B. A. Dwamena, M. Petrou et al., "Diagnostic accuracy of diffusion tensor imaging in amyotrophic lateral sclerosis: a systematic review and individual patient data metaanalysis," Academic Radiology, vol. 20, no. 9, pp. 1099-1106, 2013.
[60] M. R. Turner, M. C. Kiernan, P. N. Leigh, and K. Talbot, "Biomarkers in amyotrophic lateral sclerosis," The Lancet Neurology, vol. 8, no. 1, pp. 94-109, 2009.

[61] N. Filippini, G. Douaud, C. E. MacKay, S. Knight, K. Talbot, and M. R. Turner, "Corpus callosum involvement is a consistent feature of amyotrophic lateral sclerosis," Neurology, vol. 75, no. 18, pp. 1645-1652, 2010.

[62] M. C. Chapman, L. Jelsone-Swain, T. D. Johnson, K. L. Gruis, and R. C. Welsh, "Diffusion tensor MRI of the corpus callosum in amyotrophic lateral sclerosis," Journal of Magnetic Resonance Imaging, vol. 39, no. 3, pp. 641-647, 2013.

[63] O. Abe, H. Yamada, Y. Masutani et al., "Amyotrophic lateral sclerosis: diffusion tensor tractography and voxel-based analysis," NMR in Biomedicine, vol. 17, no. 6, pp. 411-416, 2004.

[64] O. Ciccarelli, T. E. Behrens, D. R. Altmann et al., "Probabilistic diffusion tractography: a potential tool to assess the rate of disease progression in amyotrophic lateral sclerosis," Brain, vol. 129, no. 7, pp. 1859-1871, 2006.

[65] S. Aoki, N. K. Iwata, Y. Masutani et al., "Quantitative evaluation of the pyramidal tract segmented by diffusion tensor tractography: feasibility study in patients with amyotrophic lateral sclerosis," Radiation Medicine, vol. 23, no. 3, pp. 195-199, 2005.

[66] J. Grosskreutz, J. Kaufmann, J. Frädrich, R. Dengler, H.-J. Heinze, and T. Peschel, "Widespread sensorimotor and frontal cortical atrophy in amyotrophic lateral sclerosis," BMC Neurology, vol. 6, no. 1, article 17, 2006.

[67] P. Bede, M. Elamin, S. Byrne et al., "Basal ganglia involvement in amyotrophic lateral sclerosis," Neurology, vol. 81, no. 24, pp. 2107-2115, 2013.

[68] P. Bede, A. L. Bokde, S. Byrne et al., "Multiparametric MRI study of ALS stratified for the C9orf72 genotype," Neurology, vol. 81, no. 4, pp. 361-369, 2013.

[69] B. R. Stanton, D. Shinhmar, M. R. Turner et al., "Diffusion tensor imaging in sporadic and familial (D90A SOD1) forms of amyotrophic lateral sclerosis," Archives of Neurology, vol. 66, no. 1, pp. 109-115, 2009.

[70] H. S. Kharbanda, D. C. Alsop, A. W. Anderson, G. Filardo, and D. B. Hackney, "Effects of cord motion on diffusion imaging of the spinal cord," Magnetic Resonance in Medicine, vol. 56, no. 2, pp. 334-339, 2006.

[71] C. A. Clark, G. J. Barker, and P. S. Tofts, "Improved reduction of motion artifacts in diffusion imaging using navigator echoes and velocity compensation," Journal of Magnetic Resonance, vol. 142, no. 2, pp. 358-363, 2000.

[72] T. Verma and J. Cohen-Adad, "Effect of respiration on the B0 field in the human spinal cord at 3T," Magnetic Resonance in Medicine, 2014.

[73] R. G. Nunes, P. Jezzard, and S. Clare, "Investigations on the efficiency of cardiac-gated methods for the acquisition of diffusion-weighted images," Journal of Magnetic Resonance, vol. 177, no. 1, pp. 102-110, 2005.

[74] H. U. Voss, R. Watts, A. M. Uluğ, and D. Ballon, "Fiber tracking in the cervical spine and inferior brain regions with reversed gradient diffusion tensor imaging," Magnetic Resonance Imaging, vol. 24, no. 3, pp. 231-239, 2006.

[75] H. Lundell, D. Barthelemy, F. Biering-Sørensen, J. CohenAdad, J. B. Nielsen, and T. B. Dyrby, "Fast diffusion tensor imaging and tractography of the whole cervical spinal cord using point spread function corrected echo planar imaging," Magnetic Resonance in Medicine, vol. 69, no. 1, pp. 144-149, 2012. 
[76] E. Spuentrup, A. Buecker, C. Koelker, R. W. Guenther, and M. Stuber, "Respiratory motion artifact suppression in duffusionweighted MR imaging of the spine," European Radiology, vol. 13, no. 2, pp. 330-336, 2003.

[77] P. Summers, P. Staempfli, T. Jaermann, S. Kwiecinski, and S. S. Kollias, "A preliminary study of the effects of trigger timing on diffusion tensor imaging of the human spinal cord," American Journal of Neuroradiology, vol. 27, no. 9, pp. 1952-1961, 2006.

[78] J. Cohen-Adad, M.-M. El Mendili, S. Lehéricy et al., "Demyelination and degeneration in the injured human spinal cord detected with diffusion and magnetization transfer MRI," NeuroImage, vol. 55, no. 3, pp. 1024-1033, 2011.

[79] J. Cohen-Adad, M. M. El Mendili, R. Morizot-Koutlidis et al., "Involvement of spinal sensory pathway in ALS and specificity of cord atrophy to lower motor neuron degeneration," Amyotrophic Lateral Sclerosis and Frontotemporal Degeneration, vol. 14, no. 1, pp. 30-38, 2013.

[80] F. Agosta, M. A. Rocca, P. Valsasina et al., "A longitudinal diffusion tensor MRI study of the cervical cord and brain in amyotrophic lateral sclerosis patients," Journal of Neurology, Neurosurgery \& Psychiatry, vol. 80, no. 1, pp. 53-55, 2009.

[81] G. Nair, J. D. Carew, S. Usher, D. Lu, X. P. Hu, and M. Benatar, "Diffusion tensor imaging reveals regional differences in the cervical spinal cord in amyotrophic lateral sclerosis," NeuroImage, vol. 53, no. 2, pp. 576-583, 2010.

[82] J. Cohen-Adad, "High-resolution DWI in brain and spinal cord with syngo RESOLVE," MAGNETOM Flash the Magazine of MRI, vol. 2, pp. 16-23, 2012.

[83] J. Finsterbusch, "Improving the performance of diffusionweighted inner field-of-view echo-planar imaging based on 2DSelective radiofrequency excitations by tilting the excitation plane," Journal of Magnetic Resonance Imaging, vol. 35, no. 4, pp. 984-992, 2012.

[84] J. B. Andre, G. Zaharchuk, E. Saritas et al., "Clinical evaluation of reduced field-of-view diffusion-weighted imaging of the cervical and thoracic spine and spinal cord," American Journal of Neuroradiology, vol. 33, no. 10, pp. 1860-1866, 2012.

[85] G. Zaharchuk, E. U. Saritas, J. B. Andre et al., "Reduced field-ofview diffusion imaging of the human spinal cord: comparison with conventional single-shot echo-planar imaging," American Journal of Neuroradiology, vol. 32, no. 5, pp. 813-820, 2011.

[86] D. A. Porter and R. M. Heidemann, "High resolution diffusionweighted imaging using readout-segmented echo-planar imaging, parallel imaging and a two-dimensional navigator-based reacquisition," Magnetic Resonance in Medicine, vol. 62, no. 2, pp. 468-475, 2009.

[87] D. C. van Essen and K. Ugurbil, "The future of the human connectome," NeuroImage, vol. 62, no. 4, pp. 2222-2231, 2012.

[88] J. A. McNab, B. L. Edlow, T. Witzel et al., "The human connectome project and beyond: initial applications of 300 mT/m gradients," NeuroImage, vol. 80, pp. 234-245, 2013.

[89] U. Ferizi, T. Schneider, E. Panagiotaki et al., "A ranking of diffusion MRI compartment models with in vivo human brain data," Magnetic Resonance in Medicine, 2013.

[90] D. C. Alexander, P. L. Hubbard, M. G. Hall et al., "Orientationally invariant indices of axon diameter and density from diffusion MRI," NeuroImage, vol. 52, no. 4, pp. 1374-1389, 2010.

[91] J. R. Korzan, M. Gorassini, D. Emery, Z. A. Taher, and C. Beaulieu, "In vivo magnetic resonance imaging of the human cervical spinal cord at 3 Tesla," Journal of Magnetic Resonance Imaging, vol. 16, no. 1, pp. 21-27, 2002.
[92] J. Cohen-Adad, W. Zhao, B. Keil et al., "7-T MRI of the spinal cord can detect lateral corticospinal tract abnormality in amyotrophic lateral sclerosis," Muscle \& Nerve, vol. 47, no. 5, pp. 760-762, 2013.

[93] J. Cohen-Adad, W. Zhao, L. L. Wald, and A. L. Oaklander, "7T MRI of spinal cord injury," Neurology, vol. 79, no. 22, pp. 22172217, 2012.

[94] J. Cohen-Adad, A. Mareyam, B. Keil, J. R. Polimeni, and L. L. Wald, "32-Channel RF coil optimized for brain and cervical spinal cord at 3 T," Magnetic Resonance in Medicine, vol. 66, no. 4, pp. 1198-1208, 2011.

[95] J. Cohen-Adad, B. Buchbinder, and A. L. Oaklander, "Cervical spinal cord injection of epidural corticosteroids: comprehensive longitudinal study including multiparametric magnetic resonance imaging," Pain, vol. 25, no. 6, pp. 653-676, 2012.

[96] W. Zhao, J. Cohen-Adad, J. R. Polimeni et al., "Nineteen-channel receive array and four-channel transmit array coil for cervical spinal cord imaging at 7T,' Magnetic Resonance in Medicine, 2013.

[97] M. Taso, A. le Troter, M. Sdika et al., "Construction of an in vivo human spinal cord atlas based on high-resolution MR images at cervical and thoracic levels: preliminary results," Magnetic Resonance Materials in Physics, Biology and Medicine, pp. 1-11, 2013.

[98] R. S. Samson, O. Ciccarelli, C. Kachramanoglou et al., "Tissue-and column-specific measurements from multiparameter mapping of the human cervical spinal cord at 3 T," NMR in Biomedicine, vol. 26, no. 12, pp. 1823-1830, 2013.

[99] M. C. Yiannakas, H. Kearney, R. S. Samson et al., "Feasibility of grey matter and white matter segmentation of the upper cervical cord in vivo: a pilot study with application to magnetisation transfer measurements," NeuroImage, vol. 63, no. 3, pp. 10541059, 2012.

[100] H. Kearney, M. C. Yiannakas, K. Abdel-Aziz et al., "Improved MRI quantification of spinal cord atrophy in multiple sclerosis," Journal of Magnetic Resonance Imaging, vol. 39, no. 3, pp. 617623, 2013.

[101] P. Valsasina, M. A. Horsfield, M. A. Rocca, M. Absinta, G. Comi, and M. Filippi, "Spatial normalization and regional assessment of cord atrophy: voxel-based analysis of cervical cord 3D T1weighted images," American Journal of Neuroradiology, vol. 33, no. 11, pp. 2195-2200, 2012.

[102] M. M. El Mendili, R. Chen, B. Tiret et al., "Validation of a semiautomated spinal cord segmentation method," Journal of Magnetic Resonance Imaging, 2014.

[103] B. B. Avants, N. J. Tustison, G. Song, P. A. Cook, A. Klein, and J. C. Gee, "A reproducible evaluation of ANTs similarity metric performance in brain image registration," Neurolmage, vol. 54, no. 3, pp. 2033-2044, 2011.

[104] C. Konrad, H. Henningsen, J. Bremer et al., "Pattern of cortical reorganization in amyotrophic lateral sclerosis: a functional magnetic resonance imaging study," Experimental Brain Research, vol. 143, no. 1, pp. 51-56, 2002.

[105] C. Tempelmann, C. Gaul, G. R. Kühnel et al., "Functional motor compensation in amyotrophic lateral sclerosis," Journal of Neurology, vol. 252, no. 8, pp. 944-952, 2005.

[106] A. Poujois, F. C. Schneider, I. Faillenot et al., "Brain plasticity in the motor network is correlated with disease progression in amyotrophic lateral sclerosis," Human Brain Mapping, vol. 34, no. 10, pp. 2391-2401, 2012. 
[107] M. D. Greicius, G. Srivastava, A. L. Reiss, and V. Menon, "Default-mode network activity distinguishes Alzheimer's disease from healthy aging: evidence from functional MRI," Proceedings of the National Academy of Sciences of the United States of America, vol. 101, no. 13, pp. 4637-4642, 2004.

[108] F. Agosta, P. Valsasina, M. Absinta et al., "Sensorimotor functional connectivity changes in amyotrophic lateral sclerosis," Cerebral Cortex, vol. 21, no. 10, pp. 2291-2298, 2011.

[109] G. Douaud, N. Filippini, S. Knight, K. Talbot, and M. R. Turner, "Integration of structural and functional magnetic resonance imaging in amyotrophic lateral sclerosis," Brain, vol. 134, no. 12, pp. 3470-3479, 2011.

[110] E. Verstraete, M. P. van den Heuvel, J. H. Veldink et al., "Motor network degeneration in amyotrophic lateral sclerosis: a structural and functional connectivity study," PLOS ONE, vol. 5, no. 10, Article ID e13664, 2010.

[111] L. M. Jelsone-Swain, B. W. Fling, R. D. Seidler, R. Hovatter, K. Gruis, and R. C. Welsh, "Reduced interhemispheric functional connectivity in the motor cortex during rest in limb-onset amyotrophic lateral sclerosis," Frontiers in Systems Neuroscience, vol. 4, article 158, 2010.

[112] F. Agosta, E. Canu, P. Valsasina et al., "Divergent brain network connectivity in amyotrophic lateral sclerosis," Neurobiology of Aging, vol. 34, no. 2, pp. 419-427, 2013.

[113] K. Kollewe, S. Körner, R. Dengler, S. Petri, and B. Mohammadi, "Magnetic resonance imaging in amyotrophic lateral sclerosis," Neurology Research International, vol. 2012, Article ID 608501, 9 pages, 2012.

[114] C. Luo, Q. Chen, R. Huang et al., "Patterns of spontaneous brain activity in amyotrophic lateral sclerosis: a resting-state fMRI study," PloS ONE, vol. 7, no. 9, Article ID e45470, 2012.

[115] C. Pohl, W. Block, J. Karitzky et al., "Proton magnetic resonance spectroscopy of the motor cortex in 70 patients with amyotrophic lateral sclerosis," Archives of Neurology, vol. 58, no. 5, pp. 729-735, 2001.

[116] P. Sarchielli, G. P. Pelliccioli, R. Tarducci et al., "Magnetic resonance imaging and $1 \mathrm{H}$-magnetic resonance spectroscopy in amyotrophic lateral sclerosis," Neuroradiology, vol. 43, no. 3, pp. 189-197, 2001.

[117] J. Suhy, R. G. Miller, R. Rule et al., "Early detection and longitudinal changes in amyotrophic lateral sclerosis by $1 \mathrm{H}$ MRSI," Neurology, vol. 58, no. 5, pp. 773-779, 2002.

[118] O. Gredal, S. Rosenbaum, S. Topp, M. Karlsborg, P. Strange, and L. Werdelin, "Quantification of brain metabolites in amyotrophic lateral sclerosis by localized proton magnetic resonance spectroscopy," Neurology, vol. 48, no. 4, pp. 878-881, 1997.

[119] E. P. Pioro, J. P. Antel, N. R. Cashman, and D. L. Arnold, "Detection of cortical neuron loss in motor neuron disease by proton magnetic resonance spectroscopic imaging in vivo," Neurology, vol. 44, no. 10, pp. 1933-1938, 1994.

[120] S. Kalra, C. C. Hanstock, W. R. W. Martin, P. S. Allen, and W. S. Johnston, "Detection of cerebral degeneration in amyotrophic lateral sclerosis using high-field magnetic resonance spectroscopy," Archives of Neurology, vol. 63, no. 8, pp. 1144$1148,2006$.

[121] C. J. Stagg, S. Knight, K. Talbot, M. Jenkinson, A. A. Maudsley, and M. R. Turner, "Whole-brain magnetic resonance spectroscopic imaging measures are related to disability in ALS," Neurology, vol. 80, no. 7, pp. 610-615, 2013.

[122] J. D. Carew, G. Nair, P. M. Andersen et al., "Presymptomatic spinal cord neurometabolic findings in SOD1-positive people at risk for familial ALS," Neurology, vol. 77, no. 14, pp. 1370-1375, 2011.

[123] H. Zhu, R. A. E. Edden, R. Ouwerkerk, and P. B. Barker, "High resolution spectroscopic imaging of GABA at 3 Tesla," Magnetic Resonance in Medicine, vol. 65, no. 3, pp. 603-609, 2011.

[124] B. R. Foerster, B. C. Callaghan, M. Petrou, R. A. E. Edden, T. L. Chenevert, and E. L. Feldman, "Decreased motor cortex $\gamma$ aminobutyric acid in amyotrophic lateral sclerosis," Neurology, vol. 78, no. 20, pp. 1596-1600, 2012.

[125] M. Waragai, Y. Takaya, and M. Hayashi, "Serial MRI and SPECT in amyotrophic lateral sclerosis: a case report," Journal of the Neurological Sciences, vol. 148, no. 1, pp. 117-120, 1997.

[126] G. Waldemar, S. Vorstrup, T. Staehelin Jensen, A. Johnsen, and G. Boysen, "Focal reductions of cerebral blood flow in amyotrophic lateral sclerosis: a [99mTc]-d,l-HMPAO SPECT study," Journal of the Neurological Sciences, vol. 107, no. 1, pp. 19-28, 1991.

[127] K. Abe, S. Yorifuji, and Y. Nishikawa, "Reduced isotope uptake restricted to the motor area in patients with amyotrophic lateral sclerosis," Neuroradiology, vol. 35, no. 6, pp. 410-411, 1993.

[128] S. Kalra and D. Arnold, "Neuroimaging in amyotrophic lateral sclerosis," Amyotrophic Lateral Sclerosis, vol. 4, no. 4, pp. 243248, 2003.

[129] M.-O. Habert, L. Lacomblez, P. Maksud, G. E. L. Fakhri, J.-F. Pradat, and V. Meininger, "Brain perfusion imaging in amyotrophic lateral sclerosis: extent of cortical changes according to the severity and topography of motor impairment," Amyotrophic Lateral Sclerosis, vol. 8, no. 1, pp. 9-15, 2007.

[130] J. M. Hoffman, J. C. Mazziotta, T. C. Hawk, and R. Sumida, "Cerebral glucose utilization in motor neuron disease," Archives of Neurology, vol. 49, no. 8, pp. 849-854, 1992.

[131] J. Hatazawa, R. A. Brooks, M. C. Dalakas, L. Mansi, and G. Di Chiro, "Cortical motor-sensory hypometabolism in amyotrophic lateral sclerosis: a PET study," Journal of Computer Assisted Tomography, vol. 12, no. 4, pp. 630-636, 1988.

[132] J. J. M. Kew, P. N. Leigh, E. D. Playford et al., "Cortical function in amyotrophic lateral sclerosis. A positron emission tomography study," Brain, vol. 116, no. 3, pp. 655-680, 1993.

[133] A. Cistaro, M. C. Valentini, A. Chiò et al., "Brain hypermetabolism in amyotrophic lateral sclerosis: a FDG PET study in ALS of spinal and bulbar onset," European Journal of Nuclear Medicine and Molecular Imaging, vol. 39, no. 2, pp. 251-259, 2012.

[134] A. Cistaro, M. Pagani, A. Montuschi et al., "The metabolic signature of C9ORF72-related ALS: FDG PET comparison with nonmutated patients," European Journal of Nuclear Medicine and Molecular Imaging, pp. 1-9, 2014.

[135] C. M. Lloyd, M. P. Richardson, D. J. Brooks, A. Al-Chalabi, and P. N. Leigh, "Extramotor involvement in ALS: PET studies with the GABA(A) ligand [11C]flumazenil," Brain, vol. 123, no. 11, pp. 2289-2296, 2000.

[136] M. R. Turner, A. Hammers, A. Al-Chalabi et al., "Distinct cerebral lesions in sporadic and 'D90A' SOD1 ALS: studies with [11C]flumazenil PET,' Brain, vol. 128, no. 6, pp. 1323-1329, 2005.

[137] S. Przedborski, V. Dhawan, D. M. Donaldson et al., "Nigrostriatal dopaminergic function in familial amyotrophic lateral sclerosis patients with and without copper/zinc superoxide dismutase mutations," Neurology, vol. 47, no. 6, pp. 1546-1551, 1996.

[138] G. D. Borasio, R. Linke, J. Schwarz et al., "Dopaminergic deficit in amyotrophic lateral sclerosis assessed with [I-123] IPT single photon emission computed tomography," Journal of Neurology Neurosurgery \& Psychiatry, vol. 65, no. 2, pp. 263-265, 1998. 
[139] M. R. Turner, E. A. Rabiner, A. Hammers et al., "[11C]WAY100635 PET demonstrates marked 5-HT1A receptor changes in sporadic ALS," Brain, vol. 128, no. 4, pp. 896-905, 2005.

[140] F. Chauveau, N. van Camp, F. Dollé et al., "Comparative evaluation of the translocator protein radioligands 11C-DPA713, 18F-DPA-714, and 11C-PK11195 in a rat model of acute neuroinflammation," Journal of Nuclear Medicine, vol. 50, no. 3, pp. 468-476, 2009.

[141] P. Corcia, C. Tauber, J. Vercoullie et al., "Molecular imaging of microglial activation in amyotrophic lateral sclerosis," PLoS ONE, vol. 7, no. 12, Article ID e52941, 2012. 

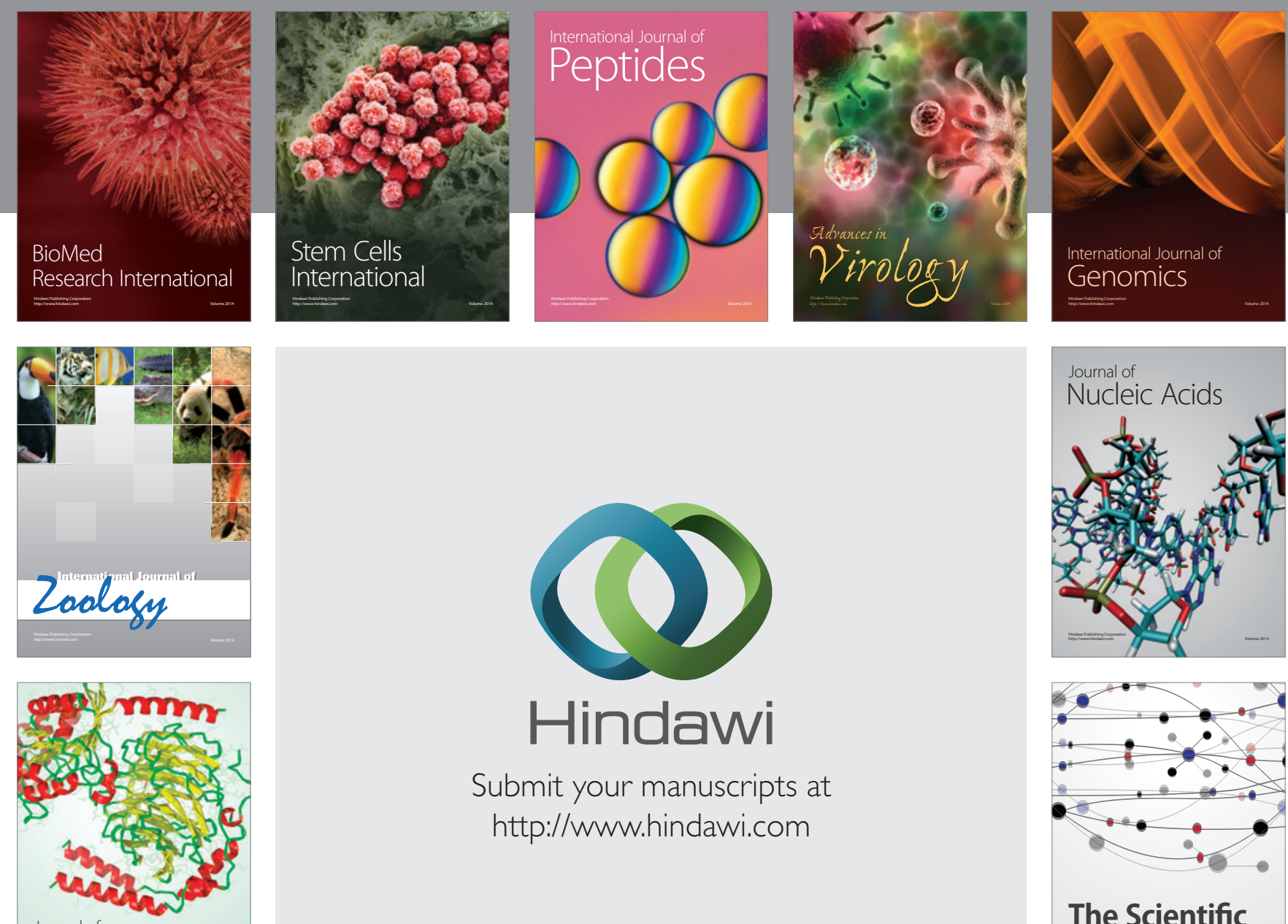

Submit your manuscripts at

http://www.hindawi.com

Journal of
Signal Transduction
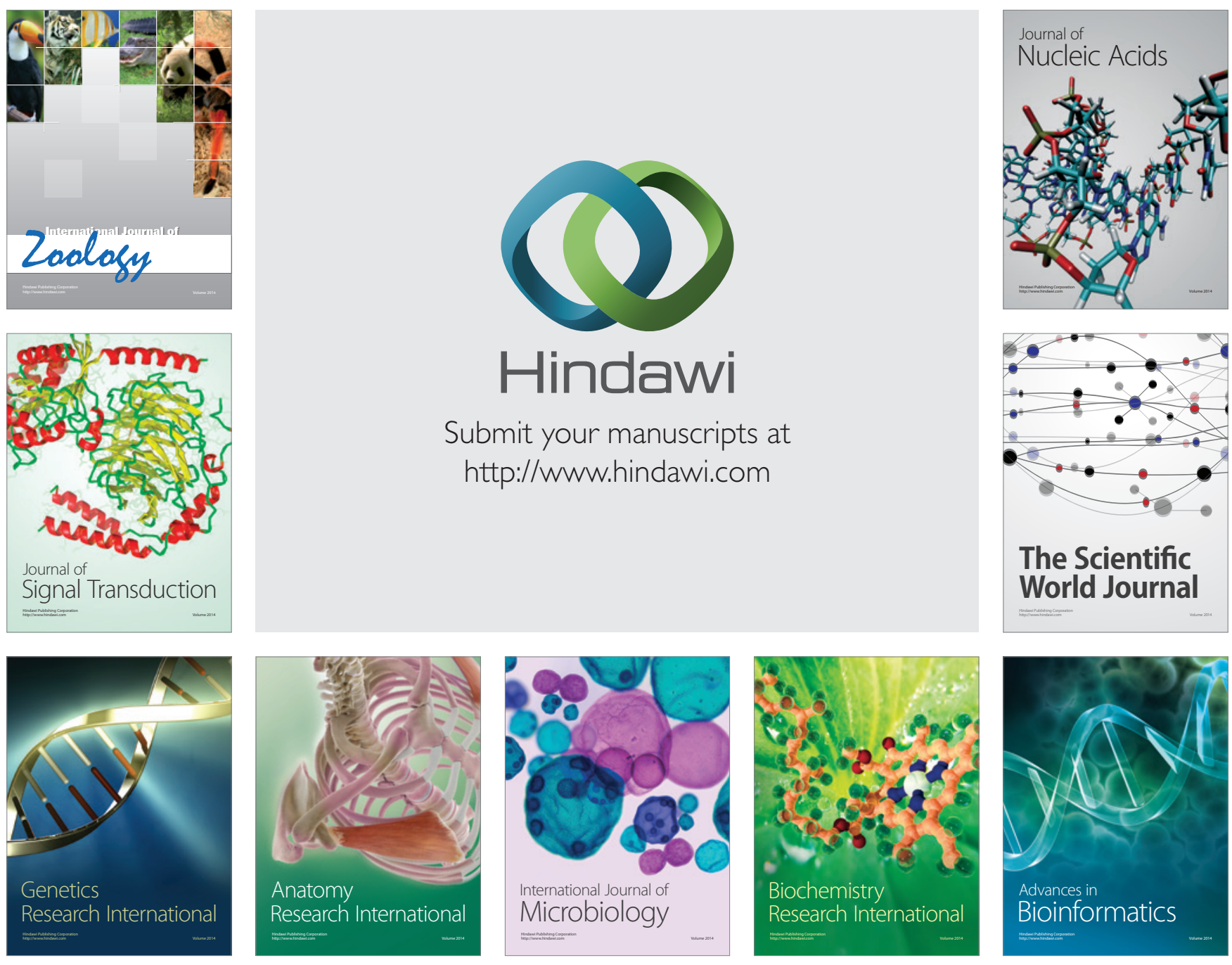

The Scientific World Journal
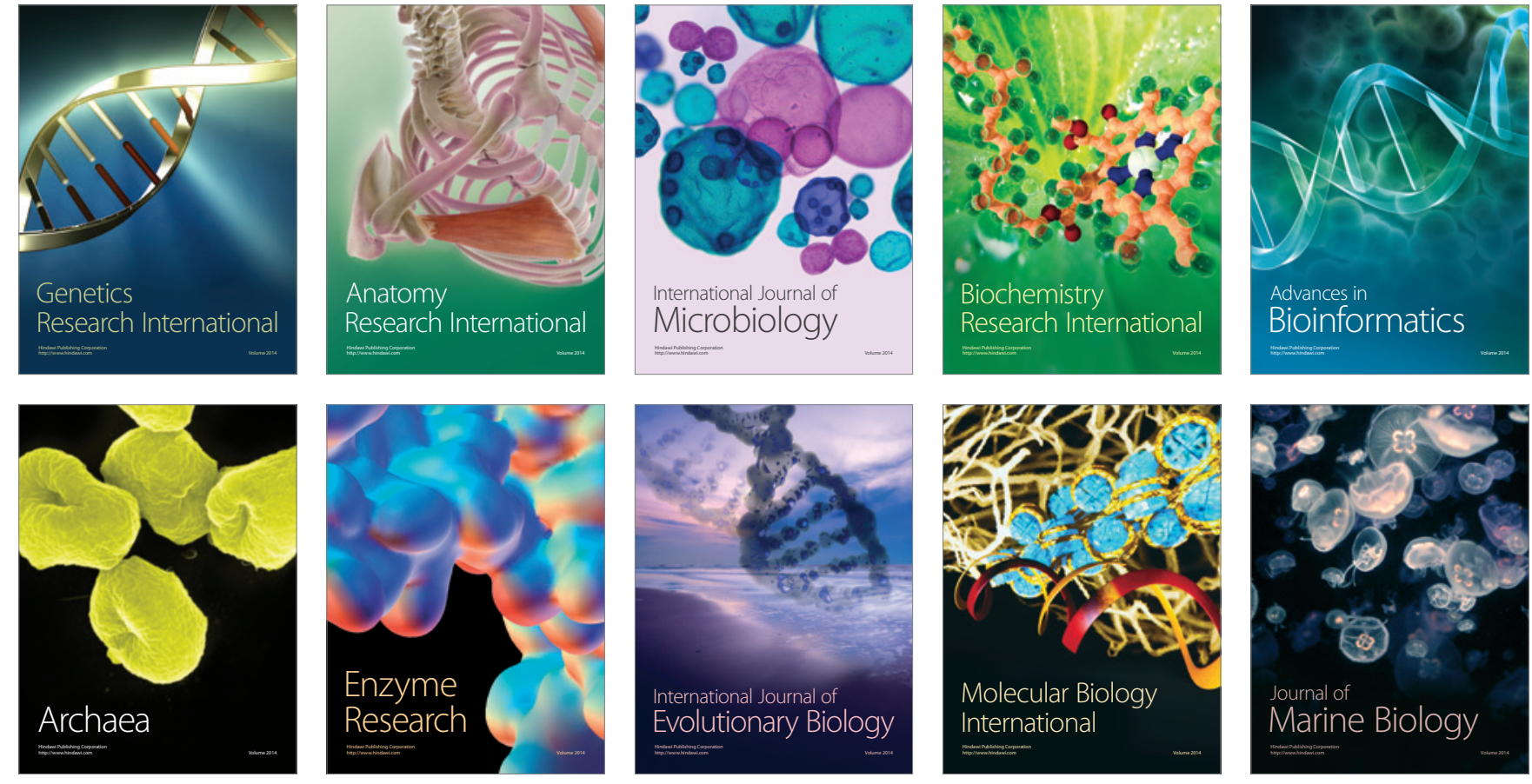